\title{
PAEDIATRIC OBSTRUCTIVE SLEEP APNEA: A REVIEW ARTICLE
}

\author{
TALUKDER DC ${ }^{1}$, MUSABBIR N $^{2}$, ISLAM MS $^{3}$
}

\begin{abstract}
:
Obstructive sleep apnea (OSA) is one of the most common causes of sleep disordered breathing in children. However, it is associated with significant morbidity, potentially impacting on long term neurocognitive and behavioural development, as well as cardiovascular outcomes and metabolic homeostasis. Diagnosis and treatment of this condition in children differs in many aspects from that in adults. In this review article, we will describe the pathophysiology, clinical features and laboratory testing and measures needed for treatment of this disease.
\end{abstract}

J Dhaka Med Coll. 2016; 25(2) : 129-133

\section{Introduction:}

Obstructive sleep apnea (OSA) is a common paediatric health problem .OSA is defined by the American Thoracic Society (ATS) ${ }^{1}$ as "a disorder of breathing during sleep characterized by prolonged partial upper airway obstruction and/or intermittent complete obstruction (obstructive apnea) that disrupts normal ventilation during sleep and normal sleep patterns."

It has been reported to affect at least $1-3 \%$ of all pre-school and school-aged children. ${ }^{2}$ It can result in severe complications if left untreated. The adverse consequences of paediatric OSA may not simply be confined to the child's immediate well-being and development, but may continue to be detrimental to the patient's long-term health in adulthood. So children at risk need to be identified, investigated and treated in a timely manner.

\section{Pathophysiology:}

The pathophysiology of OSA in childhood is likely to be a complex interaction between physiological factors such as ventilator drive and neuromuscular control, and anatomical factors such as airway structure and adenotonsillar enlargement.
Upper airway resistance increases during sleep ${ }^{31} \mathrm{t}$ with airway narrowing due to a reduction in tonic activity of the pharyngeal dilator muscles.u ${ }^{-} \mathrm{w}$ Negative pressure is generated in the upper airway during inspiration, but under normal conditions this negative pressure is balanced by the

activity of the pharyngeal dilator muscles and does not lead to collapse of the upper airway. However, in situations of increased upstream resistance such as that caused by enlargement of the upper airway lymphoid tissues in many children with OSA, collapse may occur. The adenoids, situated on the roof of the nasopharynx, enlarge from infancy through adolescence in normal children and then decrease in size during adult life. They may grow downward into the nasopharyngeal lumen, forward through the posterior choanae, or inferiorly, overlapping with the level of oropharyngeal obstruction by the tonsils.x The tonsils also begin to enlarge in early childhood. Upper airway soft tissues, including the tonsils and adenoids, grow more rapidly than

the bony structure of the nasopharynx from 3 to 5 years of age, with a consequent decrease in size of the airway during this period.

1. Dr. Debesh Chandra Talukder, Associate Professor and RS, Department of ENT \& Head-Neck Surgery, Dhaka Medical College Hospital, Dhaka.

2. Dr. Nadira Musabbir, Medical Officer, Dhaka Medical College Hospital, Dhaka.

3. Dr. Mohammad Saiful Islam, Medical Officer, Dhaka Medical College Hospital, Dhaka.

Correspondence: Dr. Debesh Chandra Talukder, Associate Professor \& RS, Department Of ENT, Dhaka Medical College Hospital, Dhaka, Tel No: 01711319296, Email: debeshchandratalukder@gmail.com 
Multiple medical conditions have emerged as being associated with an increased risk of OSA. Examples of such conditions include congenital or acquired craniofacial anomalies with fixed anatomical narrowing of the upper airway, the presence of neuromuscular dysfunction, prematurity, Down syndrome, achondroplasia, cerebral palsy, and myelomeningocele. ${ }^{2}$ In addition, and of particular importance, is the presence of obesity as a major and significant risk factor for OSA in children.y

\section{Clinical Manifestation:}

Children with OSA usually present with nocturnal as well as day-time symptoms. The most common nighttime symptoms are snoring during sleep; sometimes parents are able to describe characteristic episodic pauses in breathing despite movement of the chest or abdomen. Other descriptions include gasping, restlessness during sleep, nighttime sweating, sleeping in unusual positions, parasomnias (sleep terrors, sleep walking), and secondary nocturnal enuresis.y

Daytime symptoms of OSA are very nonspecific, but together with nighttime symptoms may help alert clinicians to clinically significant OSA: hyperactivity, difficulty concentrating/learning difficulties, behavioural difficulties, excessive daytime sleepiness, and moodiness. ${ }^{10}$ Children with OSA are often mouth breathers and sometimes have hyponasal speech. Children with severe OSA can also have growth stunting.y A history of prematurity is associated with an increased risk of OSA, and there is some evidence that a family history of OSA may also be a risk factor. ${ }^{11}$

On physical examination, findings of tonsillar hypertrophy, adenoidal facies, high arched palate, obesity, midface deficiency, macroglossia or mandibular hypoplasia, under weight or over weight may strengthen the suspicion of OSA. ${ }^{11}$ Other groups at risk for OSA are children with uncontrolled epilepsy, neuromuscular disorders, Prader-Willi syndrome, and complex medical conditions, such as achondroplasia, Chiari malformation, Ehlers-Danlos syndrome, mucopolysaccharidoses, and trisomy $21 .{ }^{11}$

\section{Diagnosis of OSA in children:}

The gold standard test for the diagnosis of OSA and assessment of its severity is an overnight, attended, in-laboratory polysomnography (sleep study). ${ }^{12}$ This is a noninvasive test involving the measurement of a number of physiologic functions overnight, typically including EEG; pulse oximetry; oronasal airflow, abdominal and chest wall movements, partial pressure of carbon dioxide (PCO2); and video recording. ${ }^{13}$ Specific pediatric measuring and scoring criteria should be used. ${ }^{13}$ Polysomnography will demonstrate the presence or absence of OSAS and also the severity of OSAS, which is helpful in planning treatment and in postoperative short and long-term management. ${ }^{1} \mathrm{t}$

Full PSGs are labour and resource intensive, requiring in hospital monitoring of the patient by skilled staff overnight, and subsequent scoring and analysis. They may also not be widely available in all countries. When PSGs are not available, possible alternatives include: nocturnal video recording, nocturnal oximetry, daytime nap polysomnography or ambulatory polysomnography. ${ }^{1} \mathrm{t}$

\section{Treatment:}

It is clear from review and other sources that OSA in children is most commonly associated with adenotonsillar hypertrophy, ${ }^{1} u$ even if obesity is now a markedly frequent occurrence, and requires a lesser degree of lymphadenoid size .The recommended initial treatment, even in obese children, consists of surgical removal of the adenoids and tonsils. However, not all children who undergo adenotonsillectomy (T\&A) for OSA are cured ${ }^{1} \mathrm{~V}$ In a meta-analysis of the published literature, the success rate for T\&A in the context of OSA was approximately $85 \% .{ }^{1} \mathrm{w}$ This figure may actually be lower, particularly among obese children with OSA, or among children with severe OSA. These findings have prompted the recommendation for repeated overnight sleep studies after adenotonsillar surgery for OSA ${ }^{1} \mathrm{X}$.

For children in whom T\&A does not lead to complete resolution of OSA, OSA related to obesity, craniofacial abnormalities, neuromuscular disorders, those who do not have significant adenotonsillar hypertrophy, or 
those who choose not to undergo surgery, positive airway pressure therapy is recommended. ${ }^{1} \mathrm{x}$ The goal is to maintain patency of the upper airway throughout the respiratory cycle, improve functional residual lung capacity and decrease work of breathing. It is important to monitor adherence to CPAP and to manage complications to optimize patient adherence. Regular long-term followup is necessary as pressure requirements will change and the interface will need to be upsized and adjusted with the growth and development of the child.

Other treatment may be indicated in certain cases. Intranasal corticosteroids have been shown to improve nasal obstruction in atopic individuals and to reduce adenoidal size and obstructive symptoms. ${ }^{1} y$ Other options that are sometimes useful in adult patients with OSA include oral appliances and devices that expand the upper airway space. However these require skilled construction and are generally efficacious in mild OSA only. However it is a viable option for those who cannot or will not use CPAP.y

Craniofacial surgery has been shown to be successful in children with syndromic craniofacial abnormalities. Success rates of 95.6\% have been reported in patients with micrognathia, ${ }^{2} \mathrm{p}$ but success rates tend to be lower in the presence of other abnormalities. Tracheostomy has the highest efficacy in the treatment of obstructive SDB when compared to other surgical interventions but is associated with worse quality of life and psychosocial development. ${ }^{21}$

\section{Consequences of paediatric OSA:}

Sleep-disordered breathing, can lead to substantial morbidities affecting the central nervous system (CNS), the cardiovascular and metabolic systems, and somatic growth, ultimately leading to reduced quality of life.

- Neurobehavioral consequences: Behavioral and neurocognitive dysfunction as well as reduced scholastic achievements are now well-characterized morbidities of OSA in children, and associations between OSA and hyperactivity and inattentive behaviors as well as cognitive deficits have been identified.$^{1} \mathrm{~V}$ In addition, parentally reported daytime sleepiness, hyperactivity, and aggressive behaviors can also develop.

The exact mechanisms by which OSA elicits such neural deficits remain relatively unresolved. Most likely, both the sleep fragmentation and episodic hypoxia that characterize OSA lead to alterations within the neurochemical substrate of the prefrontal cortex with resultant executive dysfunction, and may also elicit neuronal cell losses. ${ }^{1} \mathrm{~V}$

- Cardiovascular consequences: Similar to adult OSA, pediatric OSA has been now associated with a higher risk for cardiovascular morbidities, albeit with reduced severity of these manifestations, most likely the corollary of the increased compensatory vascular capacitance in children. For example, increased prevalence of altered blood pressure regulation, systemic hypertension, and changes in left ventricular geometry have all now been reported in children with OSA, and appear to be dose dependent. ${ }^{1} \mathrm{v}$ The mechanisms mediating cardiac and blood pressure changes are most likely associated with the increases in sympathetic activity and reactivity that progressively develop in the context of OSA.

- Quality of life and depression: Sleep disturbance associated with OSA will increase fatigue and lead to increased irritability, depressed mood, impaired concentration, and decreased interest in daily activities, and that these impairments in daily functioning may in turn interfere with other aspects of the child's life, including relationships with family, school, and peers ${ }^{22}$.

- Excessive daytime sleepiness: The exact prevalence of excessive daytime sleepiness (EDS) in pediatric OSA is unclear, and likely secondary to the overall impaired perception of caretakers, both as surrogate reporters of EDS, and also because children are unlikely to verbalize such symptoms. Several studies have thus far examined 
this particular issue. Parental reports of children being evaluated for suspected OSAS initially indicated that only a small minority of these children $(7 \%)$ presented with symptoms compatible with EDS, a rather surprising finding that would implicitly suggest that children would be relatively "protected" from OSA-induced EDS. ${ }^{1} \mathrm{~V}$

- Insulin resistance, type 2 diabetes, and metabolic syndrome: In young children, both insulin resistance (measured on the basis of the insulin:glucose ratio and homeostatic model assessment) and altered lipidemia (evidence of increased plasma triglycerides and decreased plasma high density lipoprotein concentrations) appear to be determined by the degree of obesity, and the contribution of OSA does not seem to be a major one. However, similar to adults, when obesity and OSA coincide in children the risk for metabolic disturbances is further increased. ${ }^{23}$

- Somatic growth impairment: Only 5\% or less of pediatric OSA manifest with failure to thrive. Among the proposed mechanisms for somatic growth alterations in OSA, decreased levels of insulin-like growth factor-I, insulin-like growth factor- binding proteins, and possibly growth hormone release are most likely involved. ${ }^{2} \mathrm{t}$

\section{Conclusion:}

Pediatric OSA represents a major, as of yet poorly addressed public health burden with many years of potential consequences for affected individuals, their families, and society. It is possible that in some cases it heralds an underlying abnormality of upper airway that becomes manifest as OSA in later life. Treatment is simple and effective in most cases and early recognition and treatment of the disorder could provide an opportunity for cardiovascular and metabolic risk reduction in childhood which could possibly impact the health of these children not only in childhood but also in adulthood.

\section{Referneces:}

1. American Thoracic Society. Standards and indications for cardiopulmonary sleep studies in children. Am J Respir Crit Care Med. 1996; 153(2):866-878.
2. David G. Obstructive Sleep Apnea in Children: Implications for the Developing Central Nervous system. Semin Pediatr Neurol. 2008;15(2):100-106

3. Hudgel DW, Martin RJ, Johnson B. Mechanics of the respiratory system and breathing pattern during sleep in normal humans. J Appl Physiol. 1984; 56:133-7.

4. Wiegand DA, Zwillich CW, White DP. Collapsibility of the human upper airway during normal sleep. J Appl Physiol.1989; 66:1800-8.

5. Wiegand DA, Latz B, Zwillich CW. Geniohyoid muscle activity in normal men during wakefulness and sleep. J Appl Physiol. 1990; 69:1262-9.

6. Wiegand DA, Latz B, Zwillich CW. Upper airway resistance and geniohyoid muscle activity in normal men during wakefulness and sleep. J Appl Physiol. 1990; 69:1252-61.

7. Tangel DJ, Mezzanotte WS, White DP. Influence of sleep on tensor palatine EMG and upper airway resistance in normal men. J Appl Physiol. 1991; 70: 2574-81.

8. Arens R, McDonough JM, Costarino AT. Magnetic resonance imaging of the upper airway structure of children with obstructive sleep apnea syndrome. Am J Respir Crit Care Med. 2001; 164 : 698-703

9. Narag I, Mathew JI. Childhood obesity and obstructive sleep apnea. J Nutr Metab. 2012; 299: 345-352.

10. Eleonora D, Hui LT. Update on paediatric obstructive sleep apnoea. J Thorac Dis. 2016; 8(2): 224-235.

11. Kaditis AG, Alonso Alvarez ML, Boudewyns A. Obstructive sleep disordered breathing in 2- to 18-year-old children: diagnosis and management. Eur Respir J. 2016; 47:69-94.

12. Marcus CL, Chapman D, Ward SD. Clinical practice guideline: diagnosis and management of childhood obstructive sleep apnea syndrome. Pediatr. 2002; 109(4):704-712.

13. Apostolidou MT, Alexopoulos EI, Chaidas K. Obesity and persisting sleep apnea after adenotonsillectomy in Greek children. Chest. 2008; 134(6):1149-1155.

14. Marcus CL, Brooks LJ, Draper KA, Gozal D, Halbower AC, Jones J, et al. Clinical practice guideline: diagnosis and management of childhood obstructive sleep apnea syndrome. Pediatrics. 2012; 130(3): 576-584.

15. Marcus CL. Sleep-disordered breathing in children. Am J Respir Crit Care Med. 2001; 164:16-30. 
16. Oscar SC, Leila KG, Ehab D, David G. Pediatric obstructive sleep apnea: complication, management and long-term outcomes. Proc Am Thorac Soc. 2008; 5: 274-282.

17. Lipton AJ, Gozal D. Treatment of obstructive sleep apnea in children: do we really know how? Sleep Med Rev. 2003; 7:61-80.

18. Tauman R, Gulliver TE, Krishna J, MontgomeryDowns HE, O'Brien LM, Ivanenko A, Gozal D. Persistence of obstructive sleep apnea syndrome in children after adenotonsillectomy. J Pediatr. 2006; 149: 803-808.

19. Scadding GK. Corticosteroids in the treatment of pediatric allergic rhinitis. J Allergy Clin Immunol. 2001; 108: 59-64.

20. Tahiri Y, Viezel-Mathieu A, Aldekhayel S. The effectiveness of mandibular distraction in improving airway obstruction in the pediatric population. Plast Reconstr Surg. 2014;133: 352e359 e.
21. Kremer B, Botos-Kremer AI, Eckel H. Indications, complications, and surgical techniques for pediatric tracheostomies-an update. J Pediatr Surg. 2002; 37:1556-62.

22. Crabtree VM, Varni JW, Gozal D. Health-related quality of life and depressive symptoms in children with suspected sleep-disordered breathing. Sleep. 2004; 27:1131-1138.

23. Waters KA, Sitha S, O'Brien LM, Bibby S, de Torres C, Vella S, de la Eva R. Follow-up on metabolic markers in children treated for obstructive sleep apnea. Am J Respir Crit Care Med. 2006; 174:455460 .

24. Roemmich JN, Barkley JE, D'Andrea L, Nikova $\mathrm{M}$, Rogol AD, Carskadon MA,et al. Increases in overweight after adenotonsillectomy in overweight children with obstructive sleep-disordered breathing are associated with decreases in motor activity and hyperactivity. Pediatr. 2006; 117:e200-e208. 